\section{Metastasiertes Prostatakarzinom: längeres Überleben durch Hormon- plus Chemotherapie}

\author{
Männer mit metastasiertem hormonsensitivem Prostatakarzinom haben \\ bessere Überlebensaussichten, wenn der Hormonentzug mit Docetaxel \\ kombiniert wird. Den größten Gewinn haben Patienten mit hoher Tumorlast.
}

E ine Chemotherapie kommt beim EProstatakarzinom (PCa) in der Regel erst dann zum Einsatz, wenn die Erkrankung trotz Androgenentzug (ADT) fortschreitet. Bestätigen sich die Ergebnisse der E3805-Studie, könnte das bald anders werden. Patienten mit metastasierter Erkrankung, die zu Beginn einer ADT auch Docetaxel erhielten, lebten im Median fast 14 Monate länger als Patienten mit einer ADT-Monotherapie.

In der Studie waren 790 Patienten (medianes Alter 63 Jahre) randomisiert einer der beiden Strategien zugeteilt worden. Die ADT bestand aus einem GnRH-Antagonisten oder GnRH-Analogon, die Kombination beinhaltete zusätzlich sechs Zyklen Docetaxel in einer Dosis von $75 \mathrm{mg}$ pro $\mathrm{m}^{2}$ Körperoberfläche im Abstand von jeweils drei Wochen. In beiden Gruppen hatte der Großteil der Patienten eine hohe Tumorlast (viszerale Metastasen oder mindestens vier Knochenmetastasen) und einen Gleason-Score von 8 oder mehr. Das mediane Follow-up lag bei 28,9 Monaten.

Mit einer ADT plus Chemo erreichte die mediane Überlebenszeit 57,6 Monate - und damit 13,6 Monate mehr als mit alleiniger ADT (44,0 Monate). Das entsprach einem um $39 \%$ reduzierten Risiko, im Beobachtungszeitraum zu sterben.

Besonders deutlich war der Vorteil in der Gruppe der Patienten mit hoher Tumorlast: Bei ihnen war das Überleben um 17,0 Monate verlängert - von 32,2 auf 49,2 Monate. An den Folgen des Prostatakrebses starben 85 Patienten aus der Kombinations- und 114 Patienten aus der Monotherapiegruppe. Mit ADT plus Chemo dauerte es dementsprechend auch länger, bis der Krebs kastrationsresistent wurde (20,2 vs. 11,7 Monate) und bis eine klinische Progression eintrat (33,0 vs. 19,8 Monate). Einen Rückgang des PSA-Spiegels unter $0,2 \mathrm{ng} / \mathrm{ml}$ hatten nach zwölf Monaten 27,2 \% erreicht, aber nur 16,8 \% unter ADT allein.

Als Nebenwirkung der Kombinationstherapie wurden bei $2 \%$ der Patienten allergische Reaktionen vom Grad 3 oder 4 erfasst. An einer Fatigue dritten Grades litten $4 \%$ der Patienten. Diarrhö, Stomatitis, motorische und sensorische Neuropathie betrafen jeweils maximal $1 \%$. Allerdings wurde bei einem unklaren Todesfall ein möglicher Zusammenhang mit Docetaxel gesehen.

Fazit: Die Studienautoren um Christopher J. Sweeney betonen, dass durch die Docetaxelbehandlung zu Beginn der Hormontherapie „ein substanziell längeres Überleben“ erreicht wurde. Dieser Vorteil sei vor dem Hintergrund zu sehen, dass etwa ein Drittel der Patienten, die der ADT-Gruppe zugeteilt waren, nach Entwicklung einer Kastrationsresistenz ebenfalls noch Docetaxel erhalten hatten.

Dr. Beate Schumacher

Sweeney $\mathrm{CJ}$ et al. Chemohormonal therapy in metastatic hormone-sensitive prostate cancer. N Engl J Med, 2015;373:737-46.

\section{Erratum zu:}

„Abirateron bei Prostatakarzinom: längeres Leben nach Chemotherapie" von J. Neumaier in URO-NEWS 2015;19(7-8):41.

Fälschlicherweise heißt es im Titel des abgedruckten Literatur-Reviews [Ryan CJ et al. Lancet Oncol. 2015;16(2):152-60] „Abirateron bei Prostatakarzinom: "längeres Leben nach Chemotherapie". In der Studie wurde jedoch die Wirksamkeit von Abirateron bei Patienten untersucht, die zuvor noch keine Chemotherapie erhalten hatten.

Wir bitten diesen Fehler zu entschuldigen. 